\title{
Reviewer Acknowledgements for Global Journal of Health Science, Vol. 8, No. 11
}

Global Journal of Health Science wishes to acknowledge the following individuals for their assistance with peer review of manuscripts for this issue. Their help and contributions in maintaining the quality of the journal are greatly appreciated.

Global Journal of Health Science is recruiting reviewers for the journal. If you are interested in becoming a reviewer, we welcome you to join us. Please find the application form and details at http://recruitment.ccsenet.org and e-mail the completed application form to gjhs@ccsenet.org.

\section{Reviewers for Volume 8, Number 11}

\author{
Abbas Ebadi \\ Abiodun Adeniran \\ Aedan Simon Olaso \\ Aljameel Albandary \\ Asad Ali Khan Afridi \\ Ayşegül ÖZDEMİR TOPALOĞLU \\ Carlos Martin Ardila \\ Catherine L. Haggerty \\ Celia Moffat Joel Matyanga \\ Dalia Salah El-Deen El-Sedawy \\ Delfina Gabriela Ramos \\ Dongxu Fu \\ Donna Wilson \\ Ebrahim Darvishi \\ Emad Adel Shdaifat \\ Enrico Oddone \\ Esteban Ortiz-Prado \\ Evangelia Mavrikaki \\ Evanthia Sakellari \\ Evelien Broekhof \\ Francis Kariuki \\ Francisco Quereda \\ Francisco Rodenas Rigla \\ Gaia de Campora \\ Gavric Zivana \\ Hadii M Mamudu \\ Jan Chrastina \\ Jeanna M. Piper \\ Jeevan Acharya \\ Jennifer A. Johnson \\ Jose R. Cordon
}

\author{
Kartheek R. Balapala \\ Kinley Wangdi \\ Liu Liu \\ Liye Suo \\ Lizziane Kretli Winkelstroter \\ Loray Daws \\ Łukasz Tomczyk \\ Manal S. Farmawy \\ Marcel Wullschleger \\ Nima Hafezi Nejad \\ Nishith H Vayada \\ Pavlos Sarafis \\ Pedram Iranmanesh \\ Piacherski Valery \\ Prudence Manyuchi \\ Raff Calitri \\ Raywat Deonandan \\ Ru-Jeng Teng \\ Samendra Prasad Sherchan \\ Shozab Siddiq Ali \\ SULEYMAN GORPELIOGLU \\ Tan Ching Siang \\ Tracie Lee Pasold \\ Trisha Dunning \\ Usman Khan \\ Yan Ma \\ Yevgeniy Samyshkin \\ Yongmei Huang \\ Zohreh Vanaki
}

